\title{
Inverse Problem on Heat Conduction in Heterogeneous Medium
}

\author{
Albert Schwab \\ M.A. Lavrentiev Institute of Hydrodynamics, Siberian Branch, Russian Academy of Sciences, Novosibirsk, Russia \\ Email: Schwab@ngs.ru
}

Received November 10, 2013; revised December 10, 2013; accepted December 17, 2013

Copyright (C) 2014 Albert Schwab. This is an open access article distributed under the Creative Commons Attribution License, which permits unrestricted use, distribution, and reproduction in any medium, provided the original work is properly cited. In accordance of the Creative Commons Attribution License all Copyrights @ 2014 are reserved for SCIRP and the owner of the intellectual property Albert Schwab. All Copyright (C) 2014 are guarded by law and by SCIRP as a guardian.

\section{ABSTRACT}

Under consideration is a nonclassical stationary problem on heat conduction in a body with the pre-set surface temperature and heat flow. The body contains inclusions at unknown locations and with unknown boundaries. The body and inclusions have different constant thermal conductivities. The author explores the possibility of locating inclusions. The article presents an integral criterion based on which a few statements on identification of inclusions in a body are proved.

\section{KEYWORDS}

Heat Conduction; Inclusions; Defect; Heterogeneous Medium; Inverse Problem

\section{Introduction}

Under analysis is a nonclassical problem on heat conduction, with the known body's surface temperature and surface heat flow. It is assumed that the medium is heterogeneous and contains inclusions. Coefficients of heat conductivity of the medium and inclusions are assumed by constants and differ among themselves. The problem is formulated as the problem on finding heterogeneities (flaw detection problem) by overdetermined surface conditions. On the boundary surface, the condition of continuity of the temperature and heat flow is fulfilled. Such problems belong to the nonclassical problems of mathematical physics.

Stationary problems of heat conduction are described by Equation $\Delta T=0$, where $T$ is the temperature of a body and $\Delta$ is the Laplace operator. On an interface of two mediums conditions are satisfied

$$
[T]=0, \quad[W]=\left[k \frac{\partial T}{\partial n}\right]=0,
$$

where $W$ is the heat flow; $k$ is the coefficient of the thermal conductivity.

Hereinafter $k$ is the coefficient of the host medium. It follows from (1) that the normal derivative of $T$ becomes discontinuous at the boundary of two surface.

Essentially overdefined condition for the Laplace equation mean assignment of values of $T$ and $\partial T / \partial n$ on $\partial \Omega$, or, in our case, the values of $T$ and $W$ on $\partial \Omega$.

For a homogeneous medium, evidently, the essentially overdetermined conditions cannot be arbitrary, i.e., $T$ and $\partial T / \partial n=(1 / k) W$ are functionally connected on $\partial \Omega$. Let's receive conditions of coordination for the last and consequences following from them.

Consider a body with volume $\Omega$ and surface $\partial \Omega$. Let the functions

$$
A(\xi)=\iint_{\partial \Omega} a(x) R(x, \xi) \mathrm{d} S_{x}, B(\xi)=\iint_{\partial \Omega} b(x) \partial R(x, \xi) / \partial n \mathrm{~d} S_{x}
$$

be potentials of the simple layer and double layer, respectively. Here, $R(x, \xi)$ is the fundamental solution of 
the Laplace equation [1]; $a(x)$ and $b(x)$ are the densities of the layers, and $\boldsymbol{n}$ is a vector of external normal to $\partial \Omega$. It is assumed that $\partial \Omega$ is piecewise-smooth according to Lyapunov and $a(x)$ and $b(x)$ fulfill the Holder condition. Let $T(\xi)=A(\xi)-B(\xi)$, then

$$
\iint_{\partial \Omega}\left[a(x) R(x, \xi)-b(x) \partial R(x, \xi) / \partial n \mathrm{~d} S_{x}\right]= \begin{cases}T^{+}(\xi), & \xi \in \Omega^{+}, \\ T^{-}(\xi), & \xi \in \Omega^{-} .\end{cases}
$$

Here, $\Omega^{+}$and $\Omega^{-}$are, respectively, the exterior and interior of $\Omega$, the boundary surface not included. It is stated that the densities $a(x)$ and $b(x)$ are concordant if $T^{+}(\xi)$ is continuous in $\Omega$ and is equal to $b(x)$ on $\partial \Omega$. The consistency of the densities can be interpreted as correspondence of $a(x)$ and $b(x)$ to the values of a harmonic potential $T$ and its normal derivative on $\partial \Omega$ in the homogeneous medium. By the uniqueness of the Dirichlet and Neumann problem, the use of the densities $b(x)$ and $a(x)$ unequivocally recovers the concordant $a(x)$ and $b(x)$, respectively. Hereinafter, the set $(b, a)$ of the concordant densities is denoted in terms of the class $S(\partial \Omega)$.

Statement 1. The densities $b$ and $a$ are concordant, i.e. $(b, a) \in S(\partial \Omega)$ when and only when the equality below holds true

$$
\delta(\xi)=0, \xi \in \Omega^{-}
$$

where

$$
\delta(\xi)=\int_{\partial \Omega}\left[a(x) R(x, \xi)-b(x) \frac{\partial R(x, \xi)}{\partial n}\right] \mathrm{d} S_{x} .
$$

Proof. The necessity follows apparently from Green's formula for harmonic functions [1]. Proving of the sufficiency uses equivalent of Sokhotsky-Plemeli’s formula

$$
T^{+}\left(\xi_{i}\right)=A\left(\xi_{0}\right)-B\left(\xi_{0}\right)+\frac{1}{2} b\left(\xi_{0}\right), T^{-}\left(\xi_{e}\right)=A\left(\xi_{0}\right)-B\left(\xi_{0}\right)-\frac{1}{2} b\left(\xi_{0}\right),
$$

where $\xi_{0} \in \partial \Omega$, and $\xi_{i}$ and $\xi_{e}$ are the internal and external limit points relative to $\xi_{0}$, respectively. It follows from (4) that $T^{-}\left(\xi_{e}\right)=0$ from whence, considering (5), appears $T^{+}\left(\xi_{i}\right)-T^{-}\left(\xi_{e}\right)=T^{+}\left(\xi_{i}\right)=b\left(\xi_{0}\right)$ which is to be proved.

Statement 1 is similar to the theorem on boundary values of analytic function in the complex-variable function theory where conditions of continuous extension of analytical function from the closed contour to a domain are defined.

It is worth pointing at one property of the functions belonging to the class $S(\partial \Omega)$. Let $(b, a) \in S(\partial \Omega)$ and $b_{1}$ and $a_{1}$ be the values of densities, found from (3), at the boundary of an area on $\bar{\Omega}_{1} \in \Omega$; then $\left(b_{1}, a_{1}\right) \in S\left(\partial \Omega_{1}\right)$. In addition, the condition of the Neumann problem resolvability is fulfilled at $\partial \Omega$ and $\partial \Omega_{1}$, which means that the heat flows through $\partial \Omega$ and $\partial \Omega_{1}$ are zero.

Subsequently, the potentials $b(x)=T(x)$ and the flow $W(x)=k a(x)=k \partial T(x) / \partial n$ on $\partial \Omega$ are assumed known. Then, the concordance conditions (4) are written as $(T,(1 / k) W) \in S(\partial \Omega)$. For $S(\partial \Omega)$, we also use $(T, \partial T / \partial n, k) \in S(\partial \Omega)$.

Let's prove the statement following from the statement 1 .

Statement 2. Let on the boundary $\partial \Omega$ of the domain $\Omega$ with the coefficient of the thermal conductivity coefficient $k$ the temperature $T(x)$ and the heat flow $W(x) \quad(W \neq 0$ on $\partial \Omega)$ be assigned such that $(T,(1 / k) W)=(T, \partial T / \partial n, k) \in S(\partial \Omega)$. Then $\left(T,\left(1 / k^{0}\right) W\right) \notin S(\partial \Omega)$ for any $k^{0} \neq k$ it has to be executed.

Proof. On $\partial \Omega$ a function $T^{0}$ is introduced such that $T=T^{0}$ and $k \partial T / \partial n=k^{0} \partial T^{0} / \partial n$. Assume, that $\left(T^{0}, \partial T^{0} / \partial n, k^{0}\right) \in S(\partial \Omega)$, i.e., in accord with (4)

$$
\int_{\partial \Omega}\left[T^{0}(x) \frac{\partial R(x, \xi)}{\partial n}-R(x, \xi) \frac{\partial T^{0}(x, \xi)}{\partial n}\right] d S_{x}=0, \quad \xi \in \Omega^{-} . .
$$

In the same way, from the condition $(T,(1 / k) W)=(T, \partial T / \partial n, k) \in S(\partial \Omega)$ we have

$$
\int_{\partial \Omega}\left[T(x) \frac{\partial R(x, \xi)}{\partial n}-R(x, \xi) \frac{\partial T(x, \xi)}{\partial n}\right] \mathrm{d} S_{x}=0, \quad \xi \in \Omega^{-} .
$$


The flow condition $W=k(\partial T / \partial n)=k^{0}\left(\partial T^{0} / \partial n\right)$ on $\partial \Omega$ yields

$$
\frac{\partial T^{0}(x)}{\partial n}=\frac{k}{k^{0}} \frac{\partial T(x)}{\partial n} .
$$

Placing the expression above in (6) and, then, its deduction from (7), considering $T=T^{0}$ on $\partial \Omega$, produces

$$
I(\xi)=\left[1-\frac{k}{k^{0}}\right] \int_{\partial \Omega} R(x, \xi) \frac{\partial T(x)}{\partial n} \mathrm{~d} S_{x}=0, \quad \xi \in \Omega^{-} .
$$

For the simple layer potential $I(\xi)$, it appears that the external normal derivative $\partial I(\xi) / \partial n=0$. Then, according to [1] $W(x)=0, \quad x \in \partial \Omega$, i.e. we come to a contradiction with a condition the statement. That is to say, the statement has been proved.

Statement 3 (The theoremof the coefficient problem uniqueness). Let $T$ and $W$ be assigned on $\partial \Omega$ $(W \neq 0$ on $\partial \Omega$ ). Then $k$ of the medium is uniquely found from the condition $(T,(1 / k) W) \in S(\partial \Omega)$.

Proof. Inasmuch as $W=k \partial T / \partial n$, the uniform medium concordance condition (4) can be written as

$$
\int_{\partial \Omega}\left[T(x) \frac{\partial R(x, \xi)}{\partial n}-\frac{1}{k} R(x, \xi) W(x)\right] \mathrm{d} S_{x}=0, \quad \xi \in \Omega^{-} .
$$

Let there exist $k^{0} \neq k$ for which the condition (8) holds true, too. Rewrite (8) for $k^{0}$ and diminish then (8) by

$$
I_{1}(\xi)=\frac{\left(k-k^{0}\right)}{k k^{0}} \int_{\partial \Omega} R(x, \xi) W(x) \mathrm{d} S_{x}=0, \quad \xi \in \Omega^{-} .
$$

For the simple layer potential $I_{1}(\xi)$, it appears that the external normal derivative $\partial I_{1}(\xi) / \partial n=0$. Then, according to [1], $W(x)=0, \quad x \in \partial \Omega$, i.e. we come to a contradiction with a condition the statement. That is to say, the statement has been proved.

Consequence 1. If $T$ and $W \quad(W \neq 0)$ are assigned on $\partial \Omega$ and $(T,(1 / k) W) \in S(\partial \Omega)$ and $\left(T,\left(1 / k^{0}\right) W\right) \notin S(\partial \Omega)$ hold true, then $k=k^{0}$.

The condition (8) produces the formula for the coefficient of the thermal conductivity coefficient

$$
k=\frac{\int_{\partial \Omega} R(x, \xi) W(x) \mathrm{d} S_{x}}{\int_{\partial \Omega} T(x) \frac{\partial R(x, \xi)}{\partial n}} .
$$

Let's notice that in [3] conditions for determination of the thermal conductivity coefficient for a non-stationary problem of heat conductivity are received.

Based on the introduced definitions and statements, there are a few inferences for a heterogeneous medium. A heterogeneous medium is understood to be the medium containing inclusions (defects), with the conjugacy condition (1) satisfied at their boundaries. Solving the problem on an extent from $\partial \Omega$ to an inclusion boundary $\partial \Omega_{1}$ defines $T$ and $\partial T / \partial n$ on. $\partial \Omega_{1}$. The problem on the extent from $\partial \Omega$ belongs to the known problems on the harmonic extension, i.e., Cauchy problem for the Laplace equation [2]. These problems belong to conditionally correct problems of mathematical physics and have the unique solution. Geophysics has many methods of solving such problems. One of methods is offered in [3-6].

It is assumed that the condition $\left(T,\left(1 / k^{0}\right) W\right) \in S\left(\partial \Omega_{1}\right)$ is fulfilled on the boundary $\partial \Omega_{1}$ of the inclusion with $k^{0}$. This condition is assumed to be the condition for the inclusion, which means continuity of the solution inside the inclusion, i.e., the inclusion is considered as a homogenous medium. Let us prove the following statement.

Statement 4 (condition of existence of defect in a body). Let $T(x)$ and $W(x)$ be assigned on $\partial \Omega$ $(x \in \partial \Omega)$. If the body contains an inclusion $\Omega_{1}$ with the thermal conductivity coefficient $k^{0} \neq k$ ( $k$ is the coefficient of the thermal conductivity coefficient of the host medium), then $(T,(1 / k) W) \notin S(\partial \Omega)$.

Proof. Assume $(T,(1 / k) W) \in S(\partial \Omega)$. Continuing the decision from $\partial \Omega$ to $\Omega$ on $\partial \Omega_{1}$ we will find $T$, $\partial T / \partial n$ and, consequently, $W$. According to the above mentioned property of $S(\partial \Omega)$ on $\partial \Omega_{1}$, the condition $(T, \partial T / \partial n, k) \in S\left(\partial \Omega_{1}\right)$ or, which is the same kind of thing, $(T,(1 / k) W) \in S\left(\partial \Omega_{1}\right)$ is to be fulfilled. On the other hand, the solution in the inclusion is continuous, i.e. $\left(T,\left(1 / k^{0}\right) W\right) \in S\left(\partial \Omega_{1}\right)$. Thus, we have that 
$(T,(1 / k) W) \in S\left(\partial \Omega_{1}\right)$ and $\left(T,\left(1 / k^{0}\right) W\right) \in S\left(\partial \Omega_{1}\right)$ on $\partial \Omega_{1}$. Under consequence 1 , this is only possible when $k^{0}=k$, which is a contradiction a statement condition. So, the statement has been proved.

As follows from Statement 4, an inclusion as though initiates features of a field $T$; this means that in construction of the solution in $\Omega$ via the extent from $\partial \Omega$, the potential is not expressed in terms of finite functions.

The introduced definitions and proved statements allow stating the uniqueness of finding the inclusion boundary and the heat conductivity coefficient under fulfillment of the conjugacy condition (1).

Statement 5 (Theorem of the unique definition of inclusion boundary). Let in the medium $\Omega$ with $k, T$ and $W$ on $\partial \Omega$ be known $(W \neq 0$ on $\partial \Omega)$ and let $\Omega$ contain an inclusion $\Omega_{1}$ with $k^{0}\left(k \neq k^{0}\right)$. Let the solution of the problem on the extent from $\partial \Omega$ to $\partial \Omega_{1}$, i.e. define $T$ and $W(W \neq 0)$ on $\partial \Omega_{1}$. Then the condition $\left(T,\left(1 / k^{0}\right) W\right) \in S\left(\partial \Omega_{1}\right)$ uniquely defines the boundary of the inclusion $\Omega_{1}$.

Proof. We extent the solution from $\partial \Omega$ in $\Omega$. Let there be two surfaces $\partial \Omega_{1}$ and $\partial \Omega_{1}^{\prime}$ in $\Omega$, and $\left(T,\left(1 / k^{0}\right) W\right) \in S\left(\partial \Omega_{1}\right)$ and $\left(T,\left(1 / k^{0}\right) W\right) \in S\left(\partial \Omega_{1}^{\prime}\right)$ hold true at these surfaces. Below we consider three cases.

1) Let $\Omega_{1}^{\prime} \subset \Omega_{1}$, Figure 1. Assign an arbitrary function $v$ on $\partial \Omega_{1}$ and $\partial \Omega_{1}^{\prime}$. Use the value of $v$ on $\partial \Omega_{1}$ to plot a harmonic function in $\Omega_{1}$ and find $\partial v / \partial n$ on $\partial \Omega_{1}$ for this function. Likewise, assign $v$ at $\partial \Omega_{1}^{\prime}$ and find $\partial v / \partial n$ at $\partial \Omega_{1}^{\prime}$.

For the harmonic $v$ and $T$ we will write down Green's formula

$$
\int_{\partial \Omega_{1}}\left[v \frac{1}{k^{0}} W-T \frac{\partial v}{\partial n}\right] \mathrm{d} S=0 .
$$

Likewise, write Green's function for $\partial \Omega_{1}^{\prime}$

$$
\int_{\partial \Omega_{1}^{\prime}}\left[v \frac{1}{k^{0}} W-T \frac{\partial v}{\partial n}\right] \mathrm{d} S=0 .
$$

Summing up (9) and (10) yields a Green formula for the domain $\Omega_{1}-\Omega_{1}^{\prime}$

$$
\int_{\partial\left(\Omega_{1}-\Omega_{1}^{\prime}\right)}\left[v \frac{1}{k^{0}} W-T \frac{\partial v}{\partial n}\right] \mathrm{d} S=0 .
$$

On the other hand, once the solution is continuously extendable from $\partial \Omega$ in the domain $\Omega_{1}-\Omega_{1}^{\prime}$, i.e. $(T,(1 / k) W) \in S\left(\partial\left(\Omega_{1}-\Omega_{1}^{\prime}\right)\right)$, then this solution has its Green's formula, too

$$
\int_{\partial\left(\Omega_{1}-\Omega_{1}^{\prime}\right)}\left[v \frac{1}{k} W-T \frac{\partial v}{\partial n}\right] \mathrm{d} S=0 .
$$

Diminution of (12) by (11) produces

$$
\left[\frac{1}{k}-\frac{1}{k^{0}}\right] \int_{\partial\left(\Omega_{1}-\Omega_{1}^{\prime}\right)} v W \mathrm{~d} S=0
$$

The integral (13) equals zero for the arbitrary function $v$ whence it follows that $W(x)=0$ and therefore $\partial T / \partial n=0$ on $x \in \partial\left(\Omega_{1}-\Omega_{1}^{\prime}\right)$, then $T=$ const in the domain. It follow from the harmonicity of $T$ in $\Omega / \Omega_{1}^{\prime}$ that $\partial T / \partial n=0$ on $\partial \Omega$, i.e. $W=0$ on $\partial \Omega$. Thus and so, we arrive at contradiction with the condition of our statement.

2) Let $\Omega_{1} \not \subset \Omega_{1}^{\prime}, \quad \Omega_{1}^{\prime} \not \subset \Omega_{1}$ and $\Omega_{1} \cap \Omega_{1}^{\prime} \neq \emptyset$, Figure 2. It can readily be understood that $(T,(1 / k) W) \in S\left(\partial\left(\Omega-\left(\Omega_{1} \cap \Omega_{1}^{\prime}\right)\right)\right)$. Then, inasmuch as $(T,(1 / k) W) \notin S(\partial(\Omega))$, it is evident that

$(T,(1 / k) W) \notin S\left(\partial\left(\Omega_{1} \cap \Omega_{1}^{\prime}\right)\right)$, i.e., there is an inclusion inside $\Omega_{1} \cap \Omega_{1}^{\prime}$. Let this domain be denoted as $\Omega_{1}^{\prime \prime}$. For the inclusion $\Omega_{1}^{\prime \prime}$ we have $\Omega_{1}^{\prime \prime} \subset \Omega_{1}$ and $\Omega_{1}^{\prime \prime} \subset \Omega_{1}^{\prime}$. Then, $\left(T,\left(1 / k^{0}\right) W\right) \in S\left(\partial \Omega_{1}\right)$, on the one hand, and $\left(T,\left(1 / k^{0}\right) W\right) \in S\left(\partial \Omega_{1}^{\prime \prime}\right)$, on the other hand; besides, $\Omega_{1}^{\prime \prime} \subset \Omega_{1}$, which agrees with the conditions of paragraph 1 of Statement 5 . Thus we come to a contradiction.

3) Let $\Omega_{1} \cap \Omega_{1}^{\prime}=\emptyset$, Figure 3 . The domain $\Omega$ is conditionally divided into two subdomains, one containing $\Omega_{1}$, the other containing $\Omega_{1}^{\prime}$. The domains are denoted by $\Omega^{1}$ and $\Omega^{2}$, respectively. Let an inclusion be inside $\Omega^{1}$. Then $(T,(1 / k) W) \in S\left(\partial\left(\Omega-\Omega_{1}\right)\right)$. In this case, $\Omega_{1}^{\prime} \subset\left(\Omega-\Omega_{1}\right)$. Whereupon $(T,(1 / k) W) \in S\left(\partial \Omega_{1}^{\prime}\right)$ is to be fulfilled alongside with $\left(T,\left(1 / k^{0}\right) W\right) \in S\left(\partial \Omega_{1}^{\prime}\right)$ on $\partial \Omega_{1}^{\prime}$, which contradicts consequence 1 . In case that the inclusion is inside $\Omega^{2}$, the relevant considerations will result in the same contradiction. 


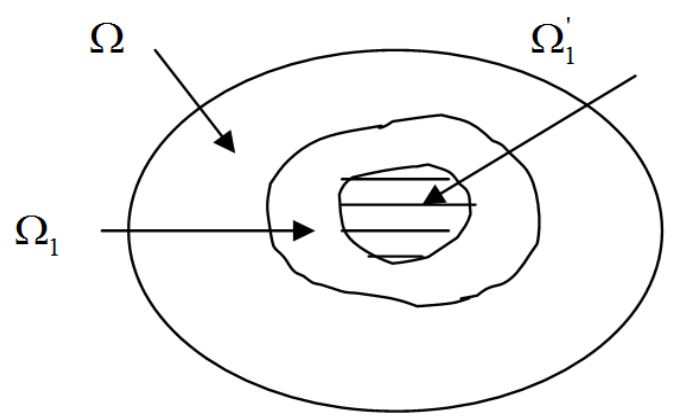

Figure 1. Conditions when one inclusion contains in other.

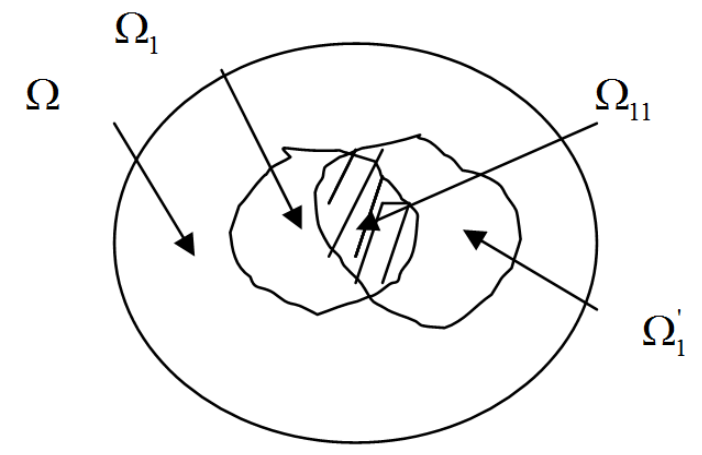

Figure 2. Conditions when two inclusions have the general area, i.e. are crossed.

$\Omega$

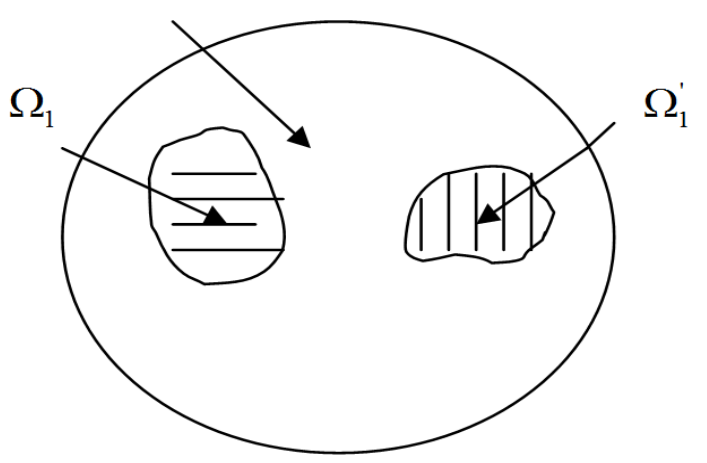

Figure 3. Conditions when inclusions aren't crossed.

With the known thermal conductivity coefficient of the host medium, it is possible to find the thermal conductivity coefficient of the inclusion.

Statement 6 (Theory of the unique definition of the thermal conductivity coefficient of inclusion). Let $T(x)$ and $W(x)$ be pre-set on $\partial \Omega(W(x) \neq 0$ at $\partial \Omega)$. Let the medium $\Omega$ with the thermal conductivity coefficient $k$ contain an inclusion $\Omega_{1}$ with the thermal conductivity coefficient $k_{1}$ and $\left(T(x), \frac{1}{k} W(x)\right) \in S\left(\partial\left(\Omega-\Omega_{1}\right)\right)$ $\left(W(x) \neq 0\right.$ on $\left.\partial \Omega_{1}\right)$. Then the condition $\left(T(x), \frac{1}{k_{1}} W(x)\right) \in S\left(\partial \Omega_{1}\right)$ uniquely defines $k_{1}$ for the inclusion.

Proof. Assume that $\Omega$ contains two surfaces $\partial \Omega_{1}$ and $\partial \Omega_{2}$ where the settings of the theorem are fulfilled and $\left(T(x), \frac{1}{k_{1}} W(x)\right) \in S\left(\partial \Omega_{1}\right),\left(T(x), \frac{1}{k_{2}} W(x)\right) \in S\left(\partial \Omega_{2}\right), k_{1} \neq k_{2} \neq k$. Likewise Statement 5 , a few cases are considered below.

1) Let $\partial \Omega_{1}=\partial \Omega_{2}$ and $k_{1} \neq k_{2}$. But if the settings are fulfilled on $\partial \Omega_{1}$ and $\partial \Omega_{2}$, then, according to con- 
sequence 1 , it must be that $k_{1}=k_{2}$. We have arrived at the contradiction.

2) Let $\partial \Omega_{1} \cap \partial \Omega_{2}=\emptyset$ Figure 3. The domain $\Omega$ is conditionally divided into two subdomains, one containing $\Omega_{1}$, the other containing $\Omega_{2}$. The domains are denoted by $\Omega^{1}$ and $\Omega^{2}$, respectively. Let an inclusion be inside $\Omega^{1}$. Accordingly, the domain $\Omega_{2}$ belongs to $\Omega-\Omega_{1}$. Under the theorem settings, $\left(T(x), \frac{1}{k} W(x)\right) \in S\left(\partial\left(\Omega-\Omega_{1}\right)\right)$, then, since $\Omega_{2} \subset\left(\Omega-\Omega_{1}\right)$, we have that $\left(T, \frac{1}{k} W(x)\right) \in S\left(\partial \Omega_{2}\right)$. On the other hand, the condition $\left(T, \frac{1}{k_{2}} W\right) \in S\left(\partial \Omega_{2}\right)$ holds true at $\Omega^{2}$, too. Then, in pursuance to consequence 1 , we get $k_{2}=k$, which is the contradiction. In case that the inclusion is inside $\Omega^{2}$, the relevant considerations will reach to the same contra-

diction. $\Omega_{1} \subset \Omega_{2}$ or $\left(\Omega_{2} \subset \Omega_{1}\right)$ Figure 1. Then $\left(T, \frac{1}{W} W(x)\right) \in S\left(\partial \Omega_{1}\right)$ and $\left(T, \frac{1}{6} W(x)\right) \in S\left(\partial \Omega_{2}\right)$. In other words, we consider the domain $\Omega_{1}$ with the inclusion $\Omega_{2}$ and $k_{1} \neq k_{2}$. Accorching to the statement 4 we come to a contradiction.

4) Let $\Omega_{1} \not \subset \Omega_{2}, \Omega_{1}^{\prime} \not \subset \Omega_{1}$ and $\Omega_{1} \cap \Omega_{2} \neq \emptyset$, Figure 2. Continuing the decision with the $\partial \Omega$ on $\partial\left(\Omega-\left(\Omega_{1} \cap \Omega_{2}\right)\right)$ we have $(T,(1 / k) W) \in S\left(\partial\left(\Omega-\left(\Omega_{1} \cap \Omega_{2}\right)\right)\right)$. Then according to the statement 4 inclusion is in $\Omega_{1} \cap \Omega_{2}$. Denoted this domain by $\Omega_{0}$. The domain $\Omega_{0}$ belongs to $\Omega_{1}$ and $\Omega_{2}$. It follows whereof that $\left(T, \frac{1}{k_{1}} W\right) \in S\left(\partial \Omega_{0}\right)$ and $\left(T, \frac{1}{k_{2}} W\right) \in S\left(\partial \Omega_{0}\right)$. According to consequence 1 , we arrive at $k_{1}=k_{2}$, which is the contradiction. The statement has been proved.

The credibility of the criterion $S(\partial \Omega)$ was tested in the two-dimensional calculations. At the side of a unit square $x, y \in[0,1]$, the values of $T$ and $W$ in a medium enclosing a circular inclusion were pre-set. The field of the inclusion was modeled by the potentials in the form of $T_{1}=C_{1} R^{-1} \sin \theta+C_{2} R \sin \theta$ and $T_{2}=C_{1} R^{-1} \cos \theta+C_{2} R \cos \theta$. The field inside the inclusion was described by $T_{1}=C_{3} R \sin \theta$ or $T_{2}=C_{3} R \cos \theta$, where $R=\left(\left(x-x_{0}\right)^{2}+\left(y-y_{0}\right)^{2}\right)^{0.5},\left(x_{0}, y_{0}\right)$ are coordinates of the inclusion; $\theta$ is angle between the vector $R$ and axis $0 X$. The constant $C_{3}$ were found from the conjugacy condition (1). The calculations used $C_{1}=-0.0025, C_{2}=52, k_{2} / k_{1}=10, R_{0}=0.007$, where $k_{1}$ and $k_{2}$ were the thermal conductivity coefficients of the host medium and inclusion, respectively, and $R_{0}$ was the inclusion radius. In a Figure 4 calculations for inclusion in the field $T_{1}$ and in a Figure 5 in the field $T_{2}$ are presented.

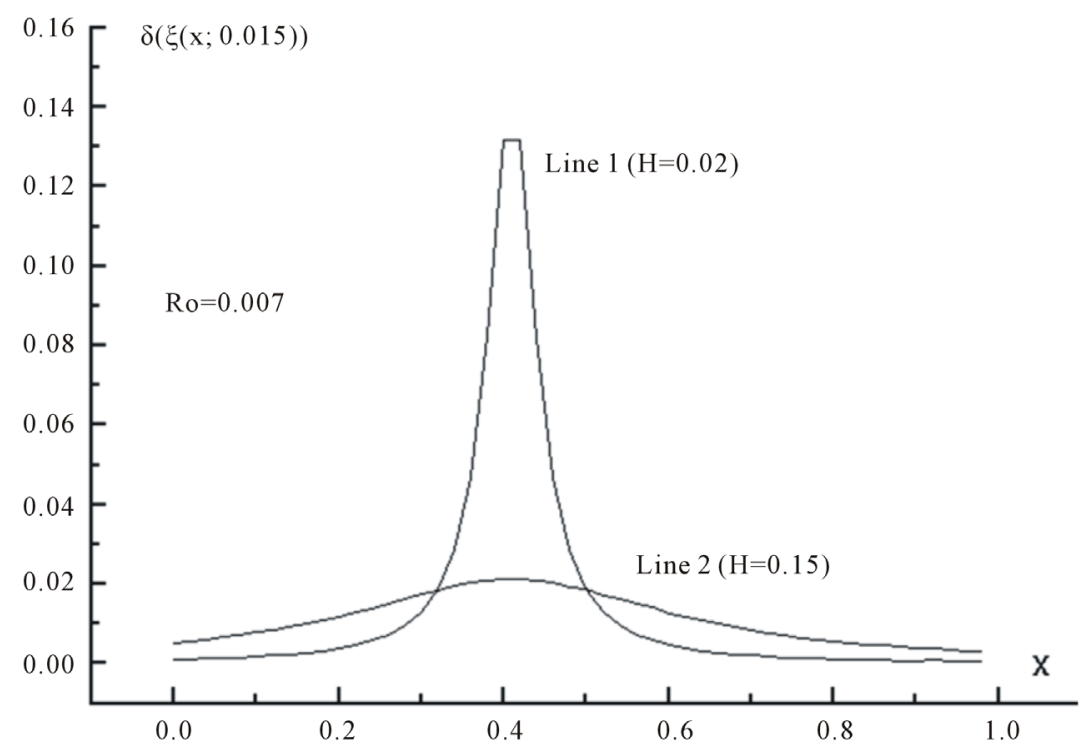

Figure 4. The curves $\delta(\xi)$ for $\xi=\xi(x ; 0.015), x \in[0,1], y=0$ in the field $T_{1}$. at the distances $H=0.02$ and $H=0.15$. 


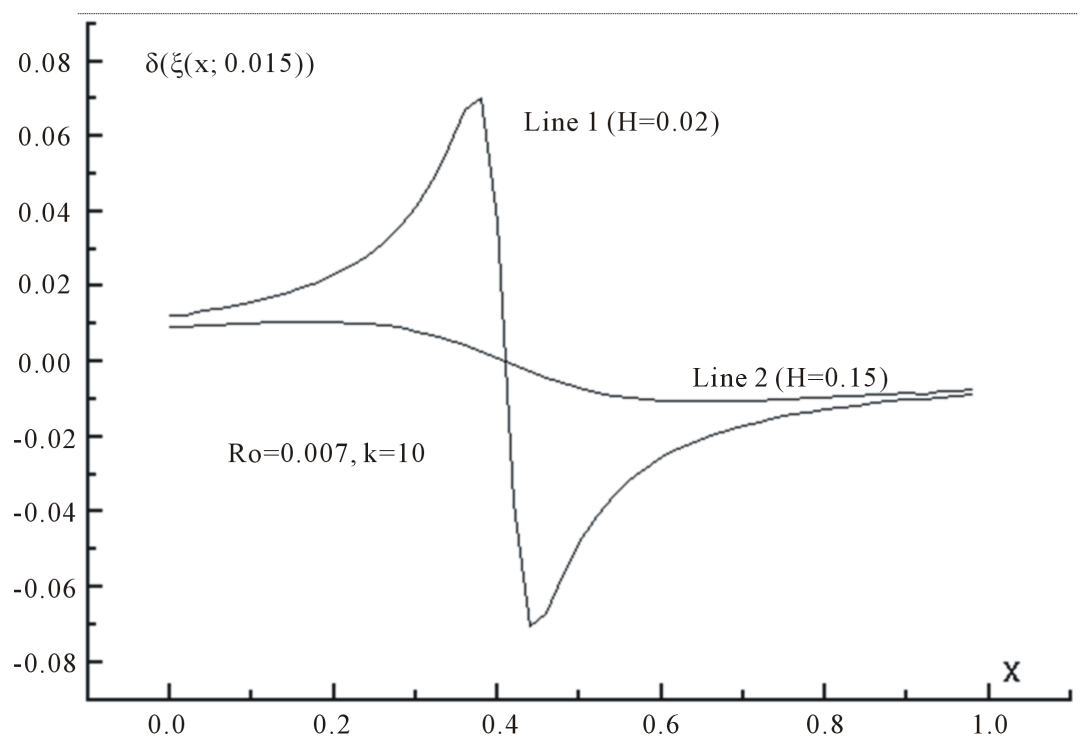

Figure 5. The curves $\delta(\xi)$ for $\xi=\xi(x ; 0.015), x \in[0,1], y=0$ in the field $T_{2}$ at the distances $H=0.02$ and $\boldsymbol{H}=\mathbf{0 . 1 5}$.

Figures 4 and 5 show the curves $\delta(\xi)$ for $\xi=\xi(x ; 0.015), x \in[0,1], y=0$, i.e. the point $\xi$ moves over the square side $y=0$ at distance 0.015 . Curves 1 and 2 correspond to the values of $\delta(\xi)$ at the distances $H=0.02$ and $H=0.15$. Values $H$ corresponds to a defect depth under the square side $y=0$.

As the calculations by the criterion $S(\partial \Omega)$ showed, the inclusion at the occurrence depth $H=0.2$ was not revealed; whereas at $R_{0}<H<0.12$ the inclusion was located at high reliability.

\section{Conclusions}

1) The criterion $S(\partial \Omega)$ allows locating inclusions in a body upon the conjugacy condition (1) at the boundary surface.

2) Based on the criterion $S(\partial \Omega)$, both the boundary of the inclusion and its thermal conductivity are uniquely defined.

3) The criterion $S(\partial \Omega)$ is reliable for near-surface inclusions.

The study was supported by the Russian Foundation for Basic Research, Project No. 11-01-00522.

\section{REFERENCES}

[1] L. S. Sobolev, “Equations of Mathematical Physics,” Nauka, Moscow, 1966.

[2] D. Lesnic, “The Determination of the Thermal Properties of Homogeneous Heat Conductors,” International Journal of Computational Methods, Vol. 1, No. 3, 2004, pp. 431-443. http://dx.doi.org/10.1142/S0219876204000228

[3] M. M. Lavrentiev, “Ill-Posed Problems in Mathematical Physics,” Novosibirsk, 1962.

[4] D. Lesnic, J. R. Berger and P. A. Martin, “A Boundary Element Regularization Method for the Boundary Determination in Potential Corrosion Damage,” Inverse Problems in Engineering, Vol. 10, No. 2, pp. 163-182.

[5] A. A. Schwab, “Computer Tomography Problem Based on the Hologram Interferometry Method,” In: Studies into Conditionally Ill-Posed Problems of Mathematical Physics, Institute of Mathematics SB AS USSR, Novosibirsk, pp. 157-162.

[6] A. A. Schwab, "Boundary Integral Equations for Inverse Problems in Elasticity Theory,” Journal of Elasticity, Vol. 41, No. 3, 1995, pp. 151-160. http://dx.doi.org/10.1007/BF00041872 